\title{
Proportional fair scheduling method with QoS for the coexistence system of GFDM and OFDM
}

\author{
Masanori Yofune, Masayuki Suto, Yasuharu Amezawa, \\ Tatsuya Yoshioka ${ }^{\text {a) }}$, and Nobuo Suzuki \\ Advanced Telecommunications Research Institute International (ATR), \\ 2-2-2 Hikaridai, Seika-cho, Soraku-gun, Kyoto 619-0288, Japan \\ a)yoshioka-tatsu@atr.jp
}

Abstract: Future 5th generation (5G) mobile system is required to accommodate a huge amount of mobile data traffic with different quality of services (QoS) in a large number of devices. To realize 5G system, a new waveform such as generalized frequency division multiplexing (GFDM) has been researched. In this paper, we propose a proportional fair (PF) scheduling method considering the delay budget of users for the coexistence system of GFDM and orthogonal frequency division multiplexing (OFDM). From computer simulation, we show that the proposed method can improve the average delay time for users of small delay budget without large degradation of system throughput.

Keywords: 5G, GFDM, OFDM, scheduling method, QoS

Classification: Wireless Communication Technologies

\section{References}

[1] 3GPP, "On coexistence of new waveforms for NR," 3GPP R1-164327, 2016.

[2] 3GPP, "Study on next generation new radio access technology," 3GPP WID RP-15922, Dec. 2015.

[3] G. Fettweis, M. Krondorf, and S. Bittner, "GFDM - generalized frequency division multiplexing,” IEEE Vehicular Technology Conference (VTC Spring), Barcelona, Spain, pp. 1-4, Apr. 2009. DOI:10.1109/VETECS.2009.5073571

[4] R. Datta, I. Kanno, and M. Ariyoshi, "Adaptive subcarrier bandwidth for 5G GFDM transmission," IEICE Technical Report, vol. 114, no. 435, SR2014-105, pp. 69-73, Jan. 2015.

[5] R. Datta, I. Kanno, and M. Ariyoshi, "Evaluation of flexible hybrid multicarrier transmission system for 5G mobile network," IEICE Technical Report, vol. 114, no. 491, SR2014-111, pp. 1-5, Mar. 2015.

[6] T. Suzuki, T. Sato, and T. Yoshioka, "Analysis of the interference from GFDM to OFDM signals in same band," IEICE Commun. Express, (Accepted in Dec. 2016). DOI:10.1587/comex.2016XBL0204

[7] C. Wengerter, J. Ohlhorst, and A. G. E. von Elbwart, "Fairness and throughput analysis for generalized proportional fair frequency scheduling in OFDMA," IEEE Vehicular Technology Conference (VTC Spring), Stockholm, Sweden, pp. 1903-1907, May 2005. DOI:10.1109/VETECS.2005.1543653 
[8] 3GPP, "Policy and charging control architecture," 3GPP TS 23.203, vol. 12.11.0, Dec. 2015.

[9] R. Datta, N. Michailow, M. Lentmaier, and G. Fettweis, "GFDM interference cancellation for flexible cognitive radio PHY design," IEEE Vehicular Technology Conference (VTC Fall), Quebec, Canada, pp. 1-5, Sept. 2012. DOI:10.1109/VTCFall.2012.6399031

\section{Introduction}

Future 5th generation (5G) mobile communication system is required to accommodate a huge amount of mobile data traffic with different quality of services (QoS) in a large number of devices. To realize 5G system, a new waveform such as generalized frequency division multiplexing (GFDM) has been researched [1, 2, 3, $4,5,6]$. In the future, if GFDM is adopted in $5 \mathrm{G}$ system, the coexistence system of GFDM and orthogonal frequency division multiplexing (OFDM) on the same system band can be considered. Since GFDM has the lower side-lobe by applying pulse shaping filter for each subcarrier, GFDM signal can be allocated to OFDM guard band. Additionally, GFDM can expect to improve the system throughput since it is flexibly available of empty resource including OFDM guard band by changing the subcarrier spacing or the number of subcarriers.

In this paper, we propose a proportional fair (PF) scheduling method considering the delay budget of each user for the coexistence system of GFDM and OFDM. The proposed method can adjust the effect of delay the budget in calculating PF metric depending on operational scenario such as the traffic amount or type of connected users. From computer simulation, we show that the proposed method can improve the average delay time for users with small delay budget without large degradation of system throughput.

\section{The coexistence system of GFDM and OFDM}

In $5 \mathrm{G}$ system, there is an amount of mobile data traffic with diversified QoS since the massive devices such as smart phones, robots and sensors are connected to the same network. In this paper, we focus on the coexistence system of GFDM and OFDM. Fig. 1 shows the allocation scenario in bandwidth of $1.4 \mathrm{MHz}$. By using GFDM, these traffics can be allocated to empty resources including guard band on the OFDM system band as shown in Fig. 1. Therefore, this system can improve the system throughput compared with the conventional OFDM system. 


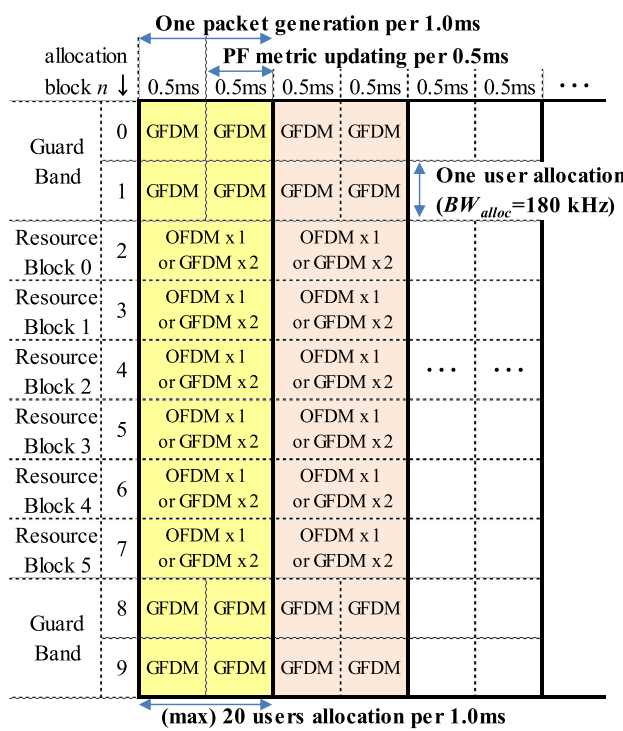

(a) Scenario 1

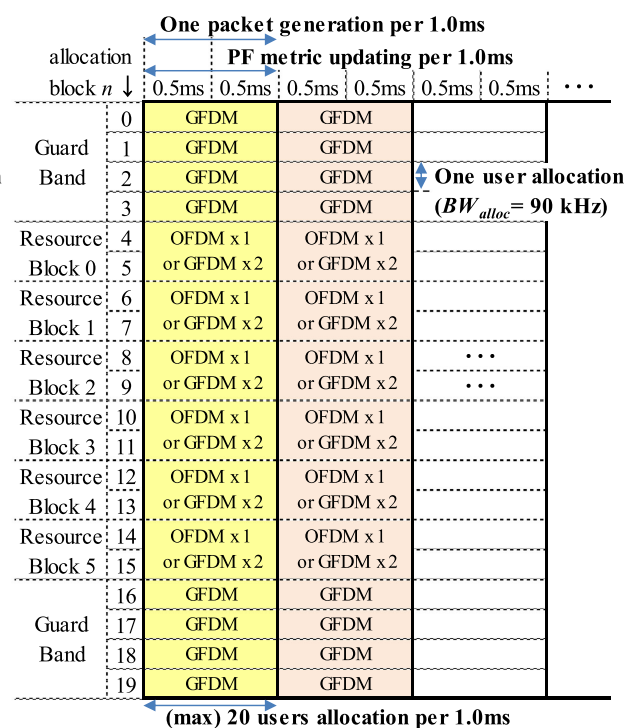

(b) Scenario2

Fig. 1. Allocation scenario at $\mathrm{BW}=1.4 \mathrm{MHz}$ of LTE.

\section{Proposed scheduling method}

The resource scheduler for $5 \mathrm{G}$ system is required not only allocating fairness between users, but also allocating based on QoS such as the delay budget. For allocation fairness, PF scheduling has been designed for $3 \mathrm{G}$ and $4 \mathrm{G}$ system [7]. In this paper, for allocation based on QoS, we propose a new PF scheduling method considering the delay budget of users for the coexistence system of GFDM and OFDM. Proposed scheduler allocates the user $x_{\text {alloc }}$ who has the largest PF metric to the allocation block $n$. The PF metric $P F(t, n, x)$ is calculated as the ratio of achievable instantaneous throughput $T P_{\text {inst }}(t, n)$ at a time $t$ and an allocation block $n$ to average throughput $T P_{\text {average }}(t)$ with correction term of the delay budget $T_{\text {delay }}(x)$ as follows:

$$
\begin{aligned}
& P F(t, n, x)=\left(\frac{T P_{\text {inst }}(t, n)}{T P_{\text {average }}(t)}\right) \cdot \frac{1}{1+\alpha \cdot\left(T_{\text {delay }}(x)\right)^{\beta}}, \\
& x_{\text {alloc }}(t, n)=\underset{X \in\left\{x_{0}, x_{1}, \ldots, x_{m}\right\}}{\arg \max }\{P F(t, n, x)\},
\end{aligned}
$$

where $m$ is the number of candidate users for allocation, $\alpha$ and $\beta$ are the weighting factors for $T_{\text {delay }}(x)$. The achievable instantaneous throughput can be calculated as follow:

$$
T P_{\text {inst }}(t, n)=B W_{\text {alloc }}(n) \cdot \log _{2}(1+\operatorname{SINR}(t, n)),
$$

where $B W_{\text {alloc }}(n)$ is the allocated bandwidth of $n, \operatorname{SINR}(t, n)$ is measured signal to interference and noise power ratio (SINR) at $t$ and $n$. In the proposed method, the weighting factors $\alpha$ and $\beta$ can adjust the effect of $T_{\text {delay }}(x)$ depending on operational scenario as the traffic amount or the type of the connected users.

\section{Performance evaluation}

In this section, the average delay time and the system throughput for the proposed scheduling method are evaluated by computer simulation with the parameters and 
Table I. Simulation parameters.

\begin{tabular}{|c|c|c|c|}
\hline User pattern name & GFDM only & OFDM only & GFDM/OFDM \\
\hline Number of GFDM user & 20 & 0 & 10 \\
\hline Number of OFDM user & 0 & 20 & 10 \\
\hline Scheduling method & \multicolumn{3}{|c|}{ Proposed PF } \\
\hline PF metric updating period [ms] & \multicolumn{3}{|c|}{0.5 (scenario1), 1.0 (scenario2) } \\
\hline Allocation BW [kHz/user] & \multicolumn{3}{|c|}{180 (scenario1), 90 (scenario2) } \\
\hline Weighting factor $\alpha$ & \multicolumn{3}{|c|}{$0.1 \sim 3$} \\
\hline Weighting factor $\beta$ & \multicolumn{3}{|c|}{$0.1 \sim 3$} \\
\hline Packet generation period [ms] & \multicolumn{3}{|c|}{1.0 (scenario1,2) } \\
\hline Traffic type & \multicolumn{3}{|c|}{ Full buffer } \\
\hline Delay budget [ms] [8] & \multicolumn{3}{|c|}{$50(\mathrm{QCI}$ no. $=3), 100(\mathrm{QCI}$ no. $=1)$} \\
\hline System bandwidth [MHz] & \multicolumn{3}{|c|}{1.4} \\
\hline Number of subcarrier & \multicolumn{3}{|c|}{128} \\
\hline Subcarrier spacing $[\mathrm{kHz}]$ & \multicolumn{3}{|c|}{15 (OFDM, GFDM) } \\
\hline GI length $[\mu \mathrm{s}]$ & \multicolumn{3}{|c|}{5.2 (OFDM, GFDM), 4.7 (OFDM) } \\
\hline Sampling frequency $[\mathrm{MHz}]$ & \multicolumn{3}{|c|}{1.92} \\
\hline Channel model & \multicolumn{3}{|c|}{ AWGN } \\
\hline Hybrid ARQ & \multicolumn{3}{|c|}{ OFF } \\
\hline ICIC (for GFDM) & \multicolumn{3}{|c|}{ ON (Double sided SIC [9]) } \\
\hline $\mathrm{AMC}$ & \multicolumn{3}{|c|}{$\mathrm{ON}$} \\
\hline Moduration & \multicolumn{3}{|c|}{ QPSK, 16QAM, 64QAM } \\
\hline FEC & \multicolumn{3}{|c|}{ Turbo Code rate $0.117 \sim 0.921$} \\
\hline Number of iteration & \multicolumn{3}{|c|}{8} \\
\hline
\end{tabular}

allocation scenarios shown in Table I and Fig. 1. We evaluate three situations that the number of OFDM users are changed. The delay time is defined as the processing time from the generation timing of a packet to the deletion timing of same packet per each user. In each user pattern of Table I, the $50 \%$ of users have the $50 \mathrm{~ms}$ delay budget, and the other users have the $100 \mathrm{~ms}$ delay budget. As shown in Fig. 1(a), scenario1 allows the allocation up to two users per $1.0 \mathrm{~ms}$ at each allocation block by updating the PF metric every $0.5 \mathrm{~ms}$. Additionally, it allows the allocation up to 10 users in $1.4 \mathrm{MHz}$ because one user is allocated per $180 \mathrm{kHz}$. On the other hand, as shown in Fig. 1(b), scenario2 allows the allocation up to one user per $1.0 \mathrm{~ms}$ at each allocation block by updating the PF metric every $1.0 \mathrm{~ms}$. In addition, it allows the allocation up to 20 users in $1.4 \mathrm{MHz}$ because of one user allocation per $90 \mathrm{kHz}$. Therefore, each scenario are able to allocate up to 20 users at $1.0 \mathrm{~ms}$ period.

Fig. 2(a-b) shows the weighting factors $\alpha$ and $\beta$ versus delay time for GFDM only pattern at scenario1. In Fig. 2(a-b), Y axis is averaged value of transmitted packets and users with same delay budget in all SNR. In Fig. 2(a), the difference of delay time between users with $50 \mathrm{~ms}$ delay budget and users with $100 \mathrm{~ms}$ delay budget is increasing in large $\alpha$. On the other hand, in Fig. 2(b), the difference of delay time between users with $50 \mathrm{~ms}$ delay budget and users with $100 \mathrm{~ms}$ delay 


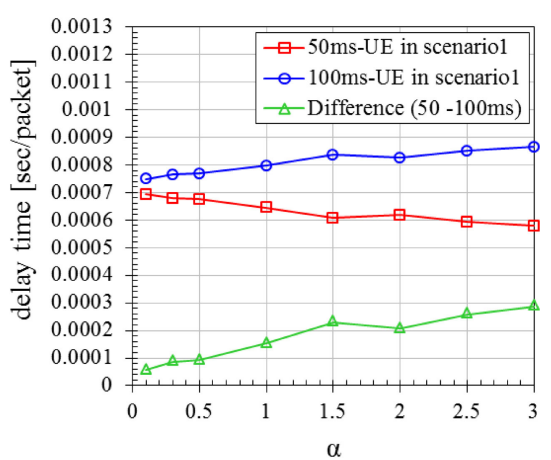

(a) Effect of weighting factor $\alpha$

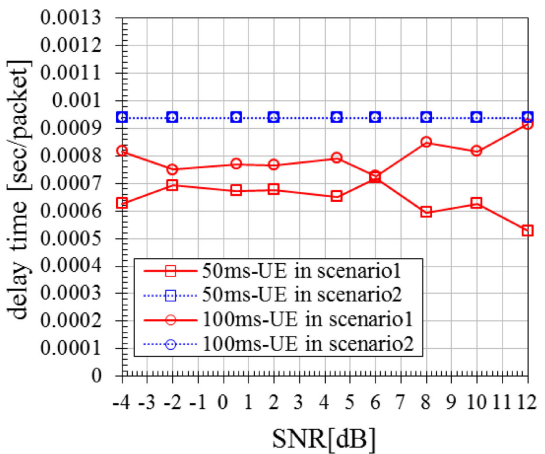

(c) Effect of multi-user allocation in time domain

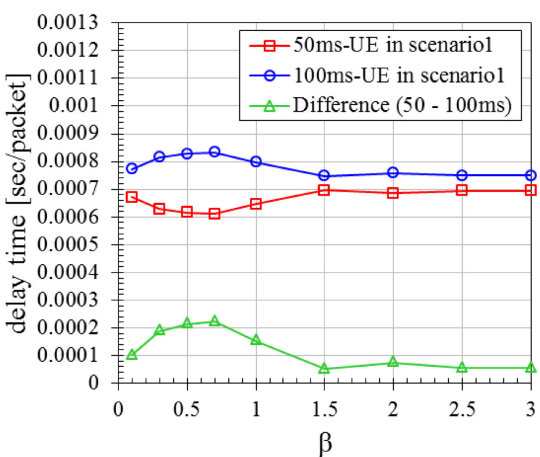

(b) Effect of weighting factor $\beta$

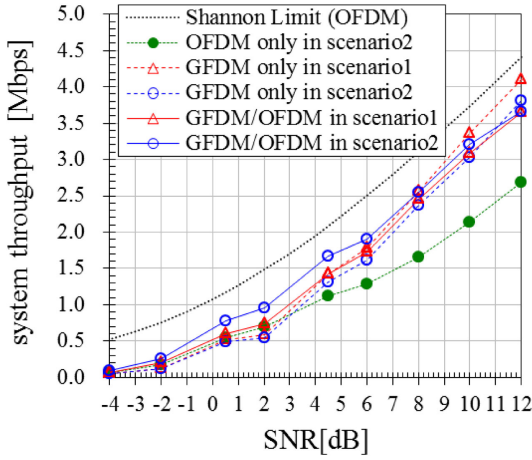

(d) system throughput

Fig. 2. Evaluation of delay time and system throughput ((a-c): for GFDM only pattern, (d): for each user pattern).

budget has the maximum value in $\beta \leqq 1$. Therefore, the proposed method can adjust the effect of delay budget by changing the values of $\alpha$ and $\beta$. In the scenario has the higher priority for the smaller delay budget, large $\alpha$ and small $\beta$ have to be set.

Fig. 2(c) shows the SNR versus delay time for GFDM only pattern in $\alpha=\beta=1$. In Fig. 2(c), Y axis is averaged value of packets and users with same delay budget in each SNR. The delay time of scenario1 is smaller than scenario2 since scenario 1 allows the allocation up to two users per $1.0 \mathrm{~ms}$. Meanwhile, the delay time of users with $50 \mathrm{~ms}$ delay budget is smaller than users with $100 \mathrm{~ms}$ delay budget. Consequently, the proposed method can preferentially allocate frequency resource to users with the small delay budget, and can improve the average delay time for these users.

Fig. 2(d) shows the SNR versus system throughput for each scenario in $\alpha=$ $\beta=1$. As shown in this figure, the system throughputs of GFDM/OFDM and GFDM only pattern at scenario2 increase about $1.0 \mathrm{Mbps}(27 \%)$ and $1.1 \mathrm{Mbps}$ $(29 \%)$ at $\mathrm{SNR}=12 \mathrm{~dB}$ compared with the OFDM only pattern, respectively. In addition, the system throughputs of GFDM/OFDM and GFDM only pattern at scenario1 increase about 1.0 Mbps (27\%) and 1.4 Mbps (34\%) compared with the OFDM only pattern. These improvements come from the guard band utilization by GFDM users.

As the above results, the proposed method can improve the average delay time for users with small delay budget without large degradation of system throughput in the same user pattern. 


\section{Conclusion}

This paper proposed the PF scheduling method considering the delay budget of each user for the coexistence system of GFDM and OFDM. The proposed method can adjust the effect of delay budget by the weighting factors $\alpha$ and $\beta$ depending on operational scenario such as the traffic amount or type of connected users. From computer simulation, we showed that the proposed method in the GFDM and OFDM coexistence system improves the average delay time for users with small delay budget without large degradation of system throughput in the coexistence system of GFDM and OFDM.

\section{Acknowledgments}

This research and development work was supported by the MIC/SCOPE \#145007107. 